\title{
Gender differences in comorbidities and risk factors in ischemic stroke patients with a history of atrial fibrillation
}

\author{
Chase Rathfoot, Camron Edrissi, Carolyn Breauna Sanders, Krista Knisely, Nicolas Poupore and Thomas Nathaniel
}

\begin{abstract}
Background: Atrial Fibrillation (AF) is a common cardiac arrhythmia and has been identified as a major risk factor for acute ischemic stroke (AIS). Gender differences in the disease process, causative mechanisms and outcomes of AF have been investigated. In the current study, we determined whether there is a gender-based disparity in AIS patients with baseline AF, and whether such a discrepancy is associated with specific risk factors and comorbidities.

Methods: Baseline factors including comorbidities, risk and demographic factors associated with a gender difference were examined using retrospective data collected from a registry from January 2010 to June 2016 in a regional stroke center. Univariate analysis was used to differentiate between genders in terms of clinical risk factors and demographics. Variables in the univariate analysis were further analyzed using logistic regression. The adjusted odds ratios (ORs) and 95\% confidence intervals ( $\mathrm{Cls}$ ) for each factor were used to predict the increasing odds of an association of a specific comorbidity and risk factor with the male or female AIS with AF.

Results: In the population of AIS patients with AF, a history of drug and alcohol use $(\mathrm{OR}=0.250,95 \% \mathrm{Cl}, 0.497-$ $1.006, P=0.016)$, sleep apnea ( $\mathrm{OR}=0.321,95 \% \mathrm{Cl}, 0.133-0.777, P=0.012)$, and higher serum creatinine ( $\mathrm{OR}=0.693$, $95 \% \mathrm{Cl}, 0.542-0.886 P=0.003)$ levels were found to be significantly associated with the male gender. Higher levels of HDL-cholesterol $(\mathrm{OR}=1.035,95 \% \mathrm{Cl}, 1.020-1.050, P<0.001)$, LDL-cholesterol $(\mathrm{OR}=1.006,95 \% \mathrm{Cl}, 1.001-1.011$, $P=0.012)$, and the inability to ambulate on admission to hospital $(\mathrm{OR}=2.258,95 \% \mathrm{Cl}, 1.368-3.727, P=0.001)$ were associated with females.

Conclusion: Our findings reveal that in the AIS patients with atrial fibrillation, migraines, HDL, LDL and poor ambulation were associated with females, while drugs and alcohol, sleep apnea, and serum creatinine level were associated with male AIS patients with AF. Further studies are necessary to determine whether gender differences in risk factor profiles and commodities require consideration in clinical practice when it comes to AF as a risk factor management in AlS patients.
\end{abstract}

Keywords: Acute ischemic stroke, Atrial fibrillation, Gender, Risk factors, Demographics

\footnotetext{
* Correspondence: nathanit@greenvillemed.sc.edu

School of Medicine Greenville, University of South Carolina, Greenville, SC 29605, USA 


\section{Introduction}

Atrial Fibrillation (AF) is a significant public health concern due to its growing prevalence and association with increased risk of cardiovascular events and death [1]. It is a common arrhythmia characterized by rapid, uncoordinated contraction of the atria, often resulting in thrombus formation and subsequent ischemia [2]. AF is a well-known independent risk factor in the development of acute ischemic stroke (AIS), as it is associated with a fivefold increase in the risk of stroke [3], and estimated to cause approximately one-fifth of all ischemic strokes, particularly of stronger severity and worse stroke-related outcomes [4]. Ischemic strokes in AF populations are associated with higher mortality, increased stroke recurrence, and greater functional deficits when compared to non-AF populations [5].

Although AF is less prevalent in females than in males, females with AF have an overall greater risk of stroke than males with AF $[6,7]$. Studies also indicate that females with AF suffer from more severe strokes [8-10]. A recent study [8] found that within the AF population, male stroke patients presented with an average NIHSS score of 6 while female stroke patients had an average score of 9 , indicating greater stroke severity and poorer functional outcomes among female stroke survivors. This finding is consistent with other studies that suggest that females with AF are more likely to suffer from debilitating or fatal strokes compared to males [3, 11, 12]. $\mathrm{AF}$ itself is a risk factor for stroke, we know that males have a higher incidence of $\mathrm{AF}$ at all age groups, while $\mathrm{AF}$ is less prevalent in females. However, females with AF have an overall greater risk of stroke than males with $\mathrm{AF}$, suggesting that comorbidities and risk factors may be more pronounced in females. However, the risk factors and comorbidities associated with the observed gender difference in AIS with baseline AF are not clear. Since female AF patients are at higher risk of stroke severity and poor functional outcomes [13], it is possible that within the population of AIS patients with baseline $\mathrm{AF}$, risk factors and comorbidities are not present in the same proportions. More risk factors and comorbidities maybe present among AF females presenting with stroke than among males. The first objective of this study is to identify the different risk factors and comorbidities in AIS population with AF and determine whether these risk factors and comorbidities are different between male and female AIS populations with a baseline AF. Since males and females do not present the same risk factors and comorbidities in the general AIS population, our second objective is to determine the association of males and females with the different comorbidities and risk factors using a retrospective data of AIS patients admitted between 2010 and 2016 to a primary stroke center. In this study, we analyzed risk factors, comorbidities and demographic variables to determine gender disparities in a stroke population with a baseline AF in a stroke center with an active patient protocol for the treatment of AIS population. Knowledge of these factors in males and females with AIS and baseline AF may necessitate different approaches to secondary prevention of stroke in patients with AF.

\section{Methods \\ Study population}

This is a population based cross sectional study for the retrospective analysis of patients' data collected from PRISMA Health ischemic stroke population. This study was approved by the PRISMA Health Ethics Committee. PRISMA Health is a primary stroke center serving patients from an 8-county region located in upper South Carolina. Data for all patients treated between January 2010 and December 2016 in the Stroke Unit of PRISMA Health were used in this study. Stroke was defined according to the World Health Organization (WHO) criteria, as "a rapidly developing clinical symptoms of focal or global disturbance of cerebral function, lasting more than $24 \mathrm{~h}$, with no apparent cause other than that of vascular origin" [14]. In this retrospective data analysis, data for ischemic stroke was based on neurologist assessment according to neuroimaging (including brain computed tomography scan and magnetic resonance imaging). Data for subtypes of stroke including subarachnoid and hemorrhagic stroke were excluded.

For each AIS patient, demographic information, risk factors, comorbidities or past medical history, and standard laboratory values were collected and analyzed. The registry has been described in previous retrospective studies [15-17]. For demographic data, information on age, race, gender, and ethnicity were retrieved in addition to their past medical and medication histories. This past medical history included data on atrial fibrillation, coronary artery disease (CAD), carotid stenosis, chronic renal disease (CRD), congestive heart failure (CHF), depression, diabetes mellitus, dyslipidemia, family history of stroke, hormone replacement therapy, hypertension, migraine, obesity, previous stroke or TIA, prosthetic heart valve, peripheral vascular disease (PVD), sleep apnea, substance use, and tobacco use. Additionally, data on site of admission (emergency department or direct admit) as well as ambulation were collected. Data on ambulation was recorded as either not documented (0), unable to ambulate (1), able to ambulate with assistance (2), and able to ambulate independently (3) at admission, during admission, and after discharge. Improvement was based on an increase in ambulation classification from admission to discharge. 


\section{Data analysis}

We conducted descriptive analyses to determine the distribution of the demographic data and risk factors in the AIS population using SPSS 24.0 (SPSS Inc. New York, New York, USA). All data have been previously normalized using the Shapiro-Wilk and Levene test to assess the data for homogeneity. Categorical variables were presented as proportions or expressed as frequencies (percentage) and quantitative variables as mean \pm standard deviation. Variables were compared between male and female patients using Pearson $\mathrm{x}^{2}$ tests to identify demographic, comorbidities, risk factors, and laboratory values associated with males or females. For ordinal and dichotomous variables (such as ambulatory status or gender), a Pearson $\mathrm{x}^{2}$ test was used while a Student's t-test was performed for all interval variables (such as age or BMI). A second univariate analysis was performed to differentiate between patients with and without a history of atrial fibrillation. Thereafter, three binary logistic multivariate analyses were performed; the first identified factors associated with gender in the whole AIS patients with and without AF. The dependent variable includes male or female patients. The second analysis focused on factors associated with males or females in the AIS population without AF, while the final analysis identified factors associated with males or females in the AIS population with baseline AF. These analyses were post-hoc adjusted logistic regression with a backward selection method. We used the backward approach because it has the advantage of considering the effects of all parameters simultaneously in fitting our regression models. This allows us to address the potential problem of multicollinearity by reducing the number of predictors and resolving the problem of overfitting. In the logistic regression, the association between the independent variables (the demographic information, comorbidities, risk factors, and laboratory values) and male or female (dependent variables) was determined for the atrial fibrillation and no atrial fibrillation group. The primary outcome is the adjusted odds ratios (ORs) and 95\% confidence intervals (CIs) for each factor associated with each gender group, fully adjusting for all confounding factors identified in our univariate analyses. The resulting ORs were used to predict the increasing odds of an association of a specific factor with the male or female AIS with or without AF. A probability value of $<0.05$ was considered statistically significant in the analyses. The HosmerLemeshow test was used to validate our model, and the overall correct classification percentage including the area under the Receiver Operating Curve (ROC) for score prediction was determined to test the sensitivity, specificity and accuracy of our logistic model.

\section{Results}

A total of 5469 AIS patients were identified. Of this, 2662 were males and 2807 were females. Table 1 presents the demographic and clinical characteristics of ischemic stroke patients stratified by male or female. As shown in Table 1, female patients were older (69.26 \pm 15.657 vs $65.13 \pm 13.372$ ) and presented with a significantly higher BMI $(28.61 \pm 7.795$ vs $28.04 \pm 5.983)$ than the male patients. For past medical history, female patients presented with significantly higher rates of atrial fibrillation (19.3\% vs $14.3 \%)$, depression (16.0\% vs 10.2 ), heart failure $(12.3 \%$ vs $9.2 \%)$, hypertension $(80.2 \%$ vs $77.2 \%)$, and migraines (3.7\% vs $1.1 \%)$. On the other hand, male patients were more likely to present with a history of coronary artery disease (35.1\% vs $25.9 \%)$, carotid artery stenosis (7.2 to $5.1 \%)$, sleep apnea (3.8\% vs $2.5 \%)$, substance use (9.6\% vs $2.9 \%)$, and tobacco use (33.2\% vs $21.5 \%)$. Regarding medication history, females were more likely to be on antihypertensives and antidepressants $(72.4 \%$ vs $66.2 \% ; 16.4 \%$ vs $9.4 \%)$ while males were more likely to be on cholesterol reducers $(46.1 \%$ vs $42.8 \%)$. Initial laboratory values also differed between male and female patients. Male patients presented with significantly higher levels of serum creatinine (1.40 \pm 1.22 vs $1.18 \pm 1.09)$ whereas female patients had higher total cholesterol $(177.54 \pm 50.89 \mathrm{mg} / \mathrm{dL}$ vs. $165.96 \pm$ $52.10 \mathrm{mg} / \mathrm{dL})$, HDL-cholesterol $(45.03 \pm 14.23 \mathrm{mg} / \mathrm{dL}$ vs. $38.43 \pm 12.57 \mathrm{mg} / \mathrm{dL})$, and LDL-cholesterol (107.31 \pm $42.58 \mathrm{mg} / \mathrm{dL}$ vs. $101.83 \pm 39.72 \mathrm{mg} / \mathrm{dL})$. Female patients also presented with a higher heart rate $(83.50 \pm 18.57$ vs $80.43 \pm 15.35)$ and a lower diastolic blood pressure $(80.39 \pm 19.59$ vs $84.60 \pm 18.37)$. Finally, a significant gender difference was observed in ambulation and stroke severity with female patients presenting with a less improved ambulation (34.3 to $37.5 \%$ ) and higher NIHSS scores (42.1\% vs $35.0 \%$ ) when compared to males.

Table 2 presents demographic and clinical characteristics of male and AIS patients stratified by the presence or absence of AF. In the AIS with no AF group, females presented with a higher age and BMI (66.66 \pm 15.581 vs $63.65 \pm 13.156 ; 29.084 \pm 28.1229 \pm 6.041)$; higher rates of depression (16.6\% vs 9.9\%), hormone replacement therapy $(2.8 \%$ vs $0.0 \%)$, migraines $(4.2 \%$ vs $1.2 \%)$, previous TIAs (9.5\% vs $7.7 \%$ ); and higher laboratory values such as total cholesterol $(181.17 \pm 51.94$ vs $168.81 \pm 46.97)$, HDL $(44.94 \pm 14.44$ vs $38.45 \pm 12.60)$, and LDL (110.03 \pm 43.69 vs $104.33 \pm 40.11)$.In addition, females presented with a higher rate for the use of antihypertensive and antidepressants $(68.3 \%$ vs $62.6 \% ; 16.6 \%$ vs $9.2 \%)$, and with a higher mean heart rate $(83.21 \pm 18.03$ vs $80.20 \pm 18.10)$. Male patients without atrial fibrillation presented with a higher rate of coronary artery disease $(32.1 \%$ vs $23.0 \%)$, substance $(10.4 \%$ vs $3.3 \%)$, and tobacco use (36.6\% vs $25 \%)$, higher serum creatine level $(1.39 \pm 1.21$ vs $1.18 \pm$ 
Table 1 Demographic and clinical characteristics of acute ischemic stroke patients compared by gender. Results for continuous variables are presented as Mean \pm SD, while discrete data are presented as percentage and frequency. Student T-test and Pearson's Chi-Square were used

\begin{tabular}{|c|c|c|c|}
\hline Characteristic & Male & Female & \\
\hline Number of patients & 2662 & 2807 & $P$-value \\
\hline \multicolumn{4}{|l|}{ Age Group: No. (\%) } \\
\hline$<50$ & $305(11.5)$ & $353(12.6)$ & \multirow[t]{5}{*}{$<0.001^{* a}$} \\
\hline $50-59$ & $603(22.7)$ & $393(14.0)$ & \\
\hline $60-69$ & $740(27.8)$ & $559(19.9)$ & \\
\hline $70-79$ & $592(22.2)$ & $639(22.8)$ & \\
\hline$>=80$ & $422(15.9)$ & 30.7) & \\
\hline Mean \pm SD & $65.13 \pm 13.372$ & $69.26 \pm 15.657$ & $<0.001^{* b}$ \\
\hline \multicolumn{4}{|l|}{ Race: No (\%) } \\
\hline White & $2090(78.5)$ & $2198(78.3)$ & \multirow[t]{3}{*}{0.544} \\
\hline Black & $492(18.5)$ & $510(18.2)$ & \\
\hline Other & $80(3.0)$ & $99(3.5)$ & \\
\hline Hispanic Ethnicity: No. (\%) & $38(1.4)$ & $47(1.7)$ & 0.461 \\
\hline BMI: Mean \pm SD & $28.04 \pm 5.983$ & $28.61 \pm 7.795$ & $0.002^{* b}$ \\
\hline \multicolumn{4}{|l|}{ Medical History: No. (\%) } \\
\hline Atrial Fib & $381(14.3)$ & $543(19.3)$ & $<0.001^{* a}$ \\
\hline Coronary Artery Disease & $935(35.1)$ & $726(25.9)$ & $<0.001^{* a}$ \\
\hline Carotid Artery Stenosis & $191(7.2)$ & $143(5.1)$ & $0.001^{* a}$ \\
\hline Depression & $271(10.2)$ & $450(16.0)$ & $<0.001^{* a}$ \\
\hline Diabetes & $951(35.7)$ & $984(35.1)$ & 0.605 \\
\hline Drugs or Alcohol & $256(9.6)$ & $81(2.9)$ & $<0.001^{* a}$ \\
\hline Dyslipidemia & $1364(51.2)$ & 1391 (49.6) & 0.213 \\
\hline Stroke Family History & $224(8.4)$ & $270(9.6)$ & 0.121 \\
\hline Heart Failure & $245(9.2)$ & $345(12.3)$ & $<0.001^{* a}$ \\
\hline Hypertension & $2056(77.2)$ & $2250(80.2)$ & $0.008^{* a}$ \\
\hline Migraine & $29(1.1)$ & $105(3.7)$ & $<0.001^{* a}$ \\
\hline Obesity & $1148(43.1)$ & $1163(41.4)$ & 0.205 \\
\hline Previous Stroke & $671(25.2)$ & $753(26.8)$ & 0.173 \\
\hline Previous TIA (> $24 \mathrm{~h}$ ) & $213(8.0)$ & $264(9.4)$ & 0.066 \\
\hline Prosthetic Heart Valve & $35(1.3)$ & $27(1.0)$ & 0.218 \\
\hline Peripheral Vascular Disease & $192(7.2)$ & $208(7.4)$ & 0.779 \\
\hline Chronic Renal Disease & $232(8.7)$ & $215(7.7)$ & 0.154 \\
\hline Sleep Apnea & $101(3.8)$ & $69(2.5)$ & $0.004^{* a}$ \\
\hline Smoker & $883(33.2)$ & $603(21.5)$ & $<0.001^{* a}$ \\
\hline \multicolumn{4}{|l|}{ Medication History: No (\%) } \\
\hline HTN medication & $1763(66.2)$ & $2031(72.4)$ & $<0.001^{* a}$ \\
\hline Cholesterol Reducer & $1227(46.1)$ & $1201(42.8)$ & $0.014^{* a}$ \\
\hline Diabetic Medication & $735(27.6)$ & $760(27.1)$ & 0.657 \\
\hline Antidepressant & $251(9.4)$ & $460(16.4)$ & $<0.001^{* a}$ \\
\hline \multicolumn{4}{|l|}{ Initial NIHSS Score: No (\%) } \\
\hline $0-9$ & $1677(74.0)$ & $1612(69.5)$ & \multirow[t]{2}{*}{$0.005^{* a}$} \\
\hline $10-14$ & $235(10.4)$ & $272(11.7)$ & \\
\hline
\end{tabular}


Table 1 Demographic and clinical characteristics of acute ischemic stroke patients compared by gender. Results for continuous variables are presented as Mean \pm SD, while discrete data are presented as percentage and frequency. Student T-test and Pearson's Chi-Square were used (Continued)

\begin{tabular}{|c|c|c|c|}
\hline Characteristic & Male & Female & \\
\hline $15-20$ & $218(9.6)$ & $283(12.2)$ & \\
\hline $21-25$ & $137(6.0)$ & $153(6.6)$ & \\
\hline Mean \pm SD & $7.63 \pm 7.84$ & $8.90 \pm 8.56$ & $<0.001^{* b}$ \\
\hline \multicolumn{4}{|l|}{ Lab values: Mean \pm SD } \\
\hline Total cholesterol & $165.96 \pm 52.10$ & $177.54 \pm 50.89$ & $<0.001^{* \mathrm{~b}}$ \\
\hline Triglycerides & $142.60 \pm 110.95$ & $136.78 \pm 99.16$ & 0.057 \\
\hline $\mathrm{HDL}$ & $38.43 \pm 12.57$ & $45.03 \pm 14.23$ & $<0.001^{* \mathrm{~b}}$ \\
\hline LDL & $101.83 \pm 39.72$ & $107.31 \pm 42.58$ & $<0.001^{* \mathrm{~b}}$ \\
\hline Lipids & $6.51 \pm 1.82$ & $6.54 \pm 3.11$ & 0.709 \\
\hline Blood Glucose & $146.75 \pm 78.26$ & $147.82 \pm 83.62$ & 0.626 \\
\hline Serum Creatinine & $1.40 \pm 1.22$ & $1.18 \pm 1.09$ & $<0.001^{* b}$ \\
\hline INR & $1.15 \pm 0.51$ & $1.18 \pm 1.09$ & 0.069 \\
\hline \multicolumn{4}{|l|}{ Vital Signs: Mean \pm SD } \\
\hline Heart Rate & $80.43 \pm 15.35$ & $83.50 \pm 18.57$ & $<0.001^{* b}$ \\
\hline Blood Pressure Systolic & $152.09 \pm 28.86$ & $151.57 \pm 29.74$ & 0.508 \\
\hline Blood Pressure Diastolic & $84.60 \pm 18.37$ & $80.39 \pm 19.59$ & $<0.001^{* \mathrm{~b}}$ \\
\hline \multicolumn{4}{|l|}{ Ambulation Status Prior to Event: No. (\%) } \\
\hline Ambulate Independently & $2453(92.1)$ & $2434(86.7)$ & $<0.001^{* a}$ \\
\hline Ambulate with Assistance & $74(2.8)$ & $129(4.6)$ & \\
\hline Unable to Ambulate & $79(3.0)$ & $134(4.8)$ & \\
\hline Not Documented & $56(2.1)$ & $109(3.9)$ & \\
\hline \multicolumn{4}{|l|}{ Ambulation Status on Admission: No. (\%) } \\
\hline Ambulate Independently & $727(27.3)$ & $604(21.5)$ & $<0.001^{* a}$ \\
\hline Ambulate with Assistance & $790(29.7)$ & $836(29.8)$ & \\
\hline Unable to Ambulate & $744(27.9)$ & $984(35.1)$ & \\
\hline Not Documented & $401(15.1)$ & $383(13.6)$ & \\
\hline \multicolumn{4}{|l|}{ Ambulation Status on Discharge: No. (\%) } \\
\hline Ambulate Independently & $1184(44.5)$ & $990(35.3)$ & $<0.001^{* a}$ \\
\hline Ambulate with Assistance & $846(31.8)$ & $974(34.7)$ & \\
\hline Unable to Ambulate & $429(16.1)$ & $640(22.8)$ & \\
\hline Not Documented & $203(7.6)$ & $203(7.2)$ & \\
\hline rtPA received: No. (\%) & $668(25.1)$ & $659(23.5)$ & 0.163 \\
\hline \multicolumn{4}{|l|}{ First Care Received: No. (\%) } \\
\hline Emergency Department & $2085(79.1)$ & $2212(79.4)$ & 0.806 \\
\hline Direct Admission & $550(20.9)$ & $574(20.6)$ & \\
\hline Improved Ambulation: No. (\%) & $927(37.5)$ & $898(34.4)$ & $0.019^{* a}$ \\
\hline NIHSS > 7: No. (\%) & $824(35.0)$ & $1043(42.1)$ & $<0.001^{* a}$ \\
\hline Diastolic Blood Pressure $\geq 80 \mathrm{mmHg}$ & 1576 (59.3) & $1312(46.8)$ & $<0.001^{* a}$ \\
\hline
\end{tabular}

Notes:

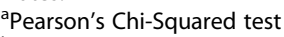

${ }^{b}$ Student's T test

* P-value < 0.05 
Table 2 Demographic and clinical characteristics of compared between atrial fibrillation status in ischemic stroke patients stratified by gender. Results for continuous variables are presented as Mean \pm SD, while discrete data are presented as percentage frequency. Student T-test and Pearson's Chi-Square were used

\begin{tabular}{|c|c|c|c|c|c|c|}
\hline \multirow[b]{2}{*}{ Characteristic } & \multicolumn{2}{|c|}{ No Atrial Fibrillation } & & \multicolumn{2}{|c|}{ With Atrial Fibrillation } & \multirow[b]{3}{*}{ P-Value } \\
\hline & Male & Female & & Male & Female & \\
\hline Number of patients & 2281 & 2264 & $P$-value & 381 & 543 & \\
\hline \multicolumn{7}{|l|}{ Age Group: No. (\%) } \\
\hline$<50$ years & 295 (12.9) & $348(15.4)$ & $<0.001^{* a}$ & $10(2.6)$ & $5(0.9)$ & $<0.001 * a$ \\
\hline $50-59$ & $568(24.9)$ & $374(16.5)$ & & $35(9.2)$ & $19(3.5)$ & \\
\hline $60-69$ & $666(29.2)$ & $499(22.0)$ & & $74(19.4)$ & $60(11.0)$ & \\
\hline $70-79$ & $468(20.5)$ & $510(22.5)$ & & $124(32.5)$ & $129(23.8)$ & \\
\hline$>=80$ & $284(12.5)$ & $533(23.5)$ & & $138(36.2)$ & $330(60.8)$ & \\
\hline Age Mean \pm SD & $63.65 \pm 13.156$ & $66.66 \pm 15.581$ & $<0.001 * b$ & $74.01 \pm 11.013$ & $80.09 \pm 10.475$ & $<0.001^{* b}$ \\
\hline \multicolumn{7}{|l|}{ Race: No (\%) } \\
\hline White & $1753(76.9)$ & $1728(76.3)$ & 0.425 & $337(88.5)$ & $470(86.6)$ & 0.692 \\
\hline Black & $452(19.8)$ & 444 (19.6) & & $40(10.5)$ & $66(12.2)$ & \\
\hline Other & $76(3.3)$ & $92(4.1)$ & & $4(1.0)$ & $7(1.3)$ & \\
\hline Hispanic Ethnicity: No. (\%) & $37(1.6)$ & $41(1.8)$ & 0.624 & $1(0.3)$ & $6(1.1)$ & 0.146 \\
\hline BMI: Mean \pm SD & $28.1229 \pm 6.041$ & $29.084 \pm 7.847$ & $<0.001^{* b}$ & $27.578 \pm 5.619$ & $26.669 \pm 7.263$ & $0.034^{* b}$ \\
\hline \multicolumn{7}{|l|}{ Medical History: No. (\%) } \\
\hline Coronary Artery Disease & $732(32.1)$ & $521(23.0)$ & $<0.001^{* a}$ & $203(53.3)$ & $205(37.8)$ & $<0.001 * a$ \\
\hline Carotid Artery Stenosis & $152(6.7)$ & $120(5.3)$ & 0.053 & $39(10.2)$ & $23(4.2)$ & $<0.001^{* a}$ \\
\hline Depression & $226(9.9)$ & $375(16.6)$ & $<0.001 * a$ & $45(11.8)$ & 75 (13.8) & 0.373 \\
\hline Diabetes & 798 (35.0) & 817 (36.1) & 0.438 & $153(40.2)$ & $167(30.8)$ & $0.003 * a$ \\
\hline Drugs or Alcohol & $238(10.4)$ & $75(3.3)$ & $<0.001^{* a}$ & $18(4.7)$ & $6(1.1)$ & $0.001 * a$ \\
\hline Dyslipidemia & $1112(48.8)$ & $1104(48.8)$ & 0.993 & $252(66.1)$ & $287(52.9)$ & $<0.001$ \\
\hline Stroke Family History & $190(8.3)$ & $216(9.5)$ & 0.152 & $34(8.9)$ & $54(9.9)$ & 0.603 \\
\hline Heart Failure & $156(6.8)$ & $187(8.3)$ & 0.070 & $89(23.4)$ & $158(29.1)$ & 0.052 \\
\hline HRT & $1(0.0)$ & $64(2.8)$ & $<0.001 * a$ & $0(0.0)$ & $14(2.6)$ & $0.002^{* a}$ \\
\hline Hypertension & $1727(75.7)$ & $1763(77.9)$ & 0.085 & $329(86.4)$ & $487(89.7)$ & 0.120 \\
\hline Migraine & $28(1.2)$ & $95(4.2)$ & $<0.001^{* a}$ & $1(0.3)$ & $10(1.8)$ & $0.029^{* a}$ \\
\hline Obesity & $982(43.1)$ & $992(43.8)$ & 0.603 & $166(43.6)$ & $171(31.5)$ & $<0.001^{* a}$ \\
\hline Previous Stroke & $559(24.5)$ & $590(26.1)$ & 0.228 & $112(29.4)$ & $163(30.0)$ & 0.839 \\
\hline Previous TIA (> 24 h) & $175(7.7)$ & $214(9.5)$ & $0.032^{* a}$ & $38(10.0)$ & $50(9.2)$ & 0.696 \\
\hline Prosthetic Heart Valve & $22(1.0)$ & $16(0.7)$ & 0.340 & $13(3.4)$ & $11(2.0)$ & 0.192 \\
\hline Peripheral Vascular Disease & $153(6.7)$ & $154(6.8)$ & 0.899 & $39(10.2)$ & $54(9.9)$ & 0.885 \\
\hline Chronic Renal Disease & $184(8.1)$ & $160(7.1)$ & 0.203 & $48(12.6)$ & $55(10.1)$ & 0.240 \\
\hline Sleep Apnea & $77(3.4)$ & $55(2.4)$ & 0.57 & $24(6.3)$ & $14(2.6)$ & $0.005^{* a}$ \\
\hline Smoker & $834(36.6)$ & $567(25.0)$ & $<0.001 * a$ & 49 (12.9) & $36(6.6)$ & $0.001^{* a}$ \\
\hline HTN medication & $1428(62.6)$ & $1546(68.3)$ & $<0.001 * a$ & $335(87.9)$ & $485(89.3)$ & 0.510 \\
\hline Cholesterol Reducer & 989 (43.4) & $951(42.0)$ & 0.357 & $238(62.5)$ & $250(46.0)$ & $<0.001^{* a}$ \\
\hline Diabetic Medication & $615(27.0)$ & $644(28.4)$ & 0.264 & $120(31.5)$ & $116(21.4)$ & $0.001 * a$ \\
\hline Antidepressant & 209 (9.2) & 375 (16.6) & $<0.001 * a$ & $42(11.0)$ & $85(15.7)$ & $0.044^{* a}$ \\
\hline \multicolumn{7}{|l|}{ Lab values: Mean \pm SD } \\
\hline Total cholesterol & $168.81 \pm 46.974$ & $181.17 \pm 51.94$ & $<0.001^{* b}$ & $148.72 \pm 73.92$ & $161.76 \pm 42.702$ & 0.002 \\
\hline Triglycerides & $146.89 \pm 114.017$ & $142.5 \pm 103.031$ & 0.205 & $116.65 \pm 85.91$ & $111.91 \pm 75.410$ & 0.414 \\
\hline $\mathrm{HDL}$ & $38.45 \pm 12.601$ & $44.94 \pm 14.437$ & $<0.001 * b$ & $38.33 \pm 12.396$ & $45.39 \pm 13.337$ & $<0.001^{* b}$ \\
\hline
\end{tabular}


Table 2 Demographic and clinical characteristics of compared between atrial fibrillation status in ischemic stroke patients stratified by gender. Results for continuous variables are presented as Mean \pm SD, while discrete data are presented as percentage frequency. Student T-test and Pearson's Chi-Square were used (Continued)

\begin{tabular}{|c|c|c|c|c|c|c|}
\hline \multirow[b]{2}{*}{ Characteristic } & \multicolumn{2}{|c|}{ No Atrial Fibrillation } & & \multicolumn{2}{|c|}{ With Atrial Fibrillation } & \\
\hline & Male & Female & & Male & Female & \\
\hline LDL & $104.33 \pm 40.106$ & $110.03 \pm 43.688$ & $<0.001^{* b}$ & $86.78 \pm 33.636$ & $95.52 \pm 35.111$ & $0.001 * b$ \\
\hline Lipids & $6.5401 \pm 1.86633$ & $6.6506 \pm 3.3761$ & 0.209 & $6.3937 \pm 1.518$ & $6.0918 \pm 1.3526$ & $0.005^{* b}$ \\
\hline Blood Glucose & $147.41 \pm 80.058$ & $150.25 \pm 87.482$ & 0.254 & $142.83 \pm 66.47$ & $137.65 \pm 64.136$ & 0.239 \\
\hline Serum Creatinine & $1.3927 \pm 1.21170$ & $1.1840 \pm 1.1746$ & $<0.001 * \mathrm{~b}$ & $1.501 \pm 1.3264$ & $1.1714 \pm 0.70412$ & $<0.001^{* b}$ \\
\hline INR & $1.0963 \pm 0.29154$ & $1.0714 \pm 0.2832$ & $0.010^{* b}$ & $1.4824 \pm 1.049$ & $1.3406 \pm 0.8496$ & $0.042^{* b}$ \\
\hline \multicolumn{7}{|l|}{ Vital Signs: Mean \pm SD } \\
\hline Heart Rate & $80.20 \pm 18.099$ & $83.21 \pm 18.034$ & $<0.001^{* b}$ & $81.79 \pm 19.790$ & $84.72 \pm 20.642$ & $0.031 * b$ \\
\hline Blood Pressure Systolic & $152.82 \pm 29.094$ & $151.69 \pm 30.417$ & 0.204 & $147.77 \pm 27.06$ & $151.04 \pm 26.749$ & 0.069 \\
\hline Blood Pressure Diastolic & $85.23 \pm 18.257$ & $80.26 \pm 19.358$ & $<0.001 * \mathrm{~b}$ & $80.84 \pm 18.633$ & $80.94 \pm 20.540$ & 0.939 \\
\hline \multicolumn{7}{|c|}{$\begin{array}{l}\text { Ambulation Status Prior to Event: } \\
\text { No. (\%) }\end{array}$} \\
\hline Ambulate Independently & $2119(92.9)$ & $2033(89.8)$ & $0.003^{* a}$ & $334(87.7)$ & 401 (73.8) & $<0.001^{* a}$ \\
\hline Ambulate with Assistance & $58(2.5)$ & $79(3.5)$ & & $16(4.2)$ & $50(9.2)$ & \\
\hline Unable to Ambulate & $65(2.8)$ & $95(4.2)$ & & $14(3.7)$ & $39(7.2)$ & \\
\hline Not Documented & $39(1.7)$ & $57(2.5)$ & & $17(4.5)$ & $52(9.6)$ & \\
\hline \multicolumn{7}{|c|}{$\begin{array}{l}\text { Ambulation Status on Admission: } \\
\text { No. }(\%)\end{array}$} \\
\hline Ambulate Independently & $660(28.9)$ & $542(23.9)$ & $<0.001 * a$ & $67(17.6)$ & $62(11.4)$ & $<0.001^{* a}$ \\
\hline Ambulate with Assistance & $671(29.4)$ & $722(31.9)$ & & $119(31.2)$ & $114(21.0)$ & \\
\hline Unable to Ambulate & $611(26.8)$ & $686(30.3)$ & & $133(34.9)$ & $298(54.9)$ & \\
\hline Not Documented & 339 (14.9) & 314 (13.9) & & $62(16.3)$ & $69(12.7)$ & \\
\hline \multicolumn{7}{|c|}{$\begin{array}{l}\text { Ambulation Status on Discharge: } \\
\text { No. (\%) }\end{array}$} \\
\hline Ambulate Independently & $1061(46.5)$ & 887 (39.2) & $<0.001 * a$ & $123(32.3)$ & $103(19.0)$ & $<0.001^{* a}$ \\
\hline Ambulate with Assistance & 718 (31.5) & $796(35.2)$ & & $128(33.6)$ & $178(32.8)$ & \\
\hline Unable to Ambulate & $338(14.8)$ & $449(19.8)$ & & $91(23.9)$ & $191(35.2)$ & \\
\hline Not Documented & $164(7.2)$ & $132(5.8)$ & & $39(10.2)$ & $71(13.1)$ & \\
\hline rtPA Administration & $584(25.6)$ & $532(23.5)$ & & & & \\
\hline \multicolumn{7}{|l|}{$\begin{array}{l}\text { First Care Received } \\
\text { No. (\%) }\end{array}$} \\
\hline Emergency Department & $1772(78.5)$ & $1767(78.7)$ & 0.895 & $313(82.8)$ & $445(82.4)$ & 0.876 \\
\hline Direct Admission & $485(21.5)$ & 479 (21.3) & & $65(17.2)$ & 95 (17.6) & \\
\hline
\end{tabular}

Notes:

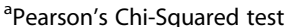

${ }^{\mathrm{b}}$ Student's T test

* $P$-value $<0.05$

1.17), INR $(1.09 \pm 0.29$ vs $1.07 \pm 0.28)$, diastolic blood pressure $(85.23 \pm 18.26$ vs $80.26 \pm 19.36)$ and ambulation improvement.

For AIS patients with a past medical history of AF, females were older $(80.09 \pm 10.48$ vs $74.01 \pm 11.01)$, use antidepressants $(15.7 \%$ vs $11.0 \%)$, HDL-cholesterol $(45.39 \pm 13.34$ vs $38.33 \pm 12.40)$, and presents with higher HRT (2.6\% vs $0.0 \%)$, migraines (1.8\% vs $0.3 \%)$, LDLcholesterol (95.52 \pm 35.11 vs $86.78 \pm 33.64)$, and heart rate $(84.72 \pm 20.64$ vs $81.79 \pm 19.79)$. Male AIS patients with AF were more likely to present with higher BMI $(27.58 \pm 5.62$ vs $26.67 \pm 7.26)$, a past medical history of coronary artery disease (53.3\% vs $37.8 \%)$, carotid artery stenosis $(10.2 \%$ vs $4.2 \%)$, diabetes $(40.2 \%$ vs $30.8 \%)$, substance use (4.7\% vs $1.1 \%)$, obesity (43.6\% vs $31.5 \%)$, sleep apnea (6.3\% vs $2.6 \%)$, tobacco use (12.9\% vs $6.6 \%)$, cholesterol reducers $(62.5 \%$ vs $46.0 \%)$, and diabetic medication (31.5\% vs $21.4 \%)$; and higher laboratory values 
including serum creatinine $(1.50 \pm 1.33$ vs $1.17 \pm 0.70)$ and INR $(1.48 \pm 1.05$ vs $1.34 \pm 0.85)$.

In the adjusted analysis for the whole AIS population with and without AF (Fig. 1), coronary artery disease $(\mathrm{OR}=0.544,95 \% \mathrm{CI}, 0.457-0.65, P<0.001)$, history of smoking $(\mathrm{OR}=0.608,95 \% \mathrm{CI}, 0.511-0.72, \mathrm{P}<0.001)$, increasing serum creatinine $(\mathrm{OR}=0.788,95 \% \mathrm{CI}, 0.712$ $0.87, P<0.001)$, increasing diastolic blood pressure $(\mathrm{OR}=0.979,95 \% \mathrm{CI}, 0.975-0.98, P=0.001)$, changes or improvement in ambulation $(\mathrm{OR}=0.829,95 \% \mathrm{CI}, 0.711-$ $0.97, P=0.017)$ were associated with males, while atrial fibrillation $(\mathrm{OR}=1.308,95 \% \mathrm{CI}, 1.051-1.63, P=0.016)$, heart failure $(\mathrm{OR}=1.468,95 \% \mathrm{CI}, 1.124-1.92, P=0.005)$, anti-HTN medication $(\mathrm{OR}=1.549,95 \% \mathrm{CI}, 1.085-2.21$, $\mathrm{P}=0.016)$, increasing total cholesterol $\mathrm{l}(\mathrm{OR}=1.005,95 \%$ CI, 1.003-1.01, $P<0.001$ ), increasing HDL-cholesterol $(\mathrm{OR}=1.037,95 \% \mathrm{CI}, 1.03-1.04, \mathrm{P}<0.001)$, and increasing NIHSS $(\mathrm{OR}=1.022,95 \% \mathrm{CI}, 1.01-1.04, P<0.001)$ were associated with females. As shown in Fig. 2, the ROC Curve (AUC $=0.729,95 \% \mathrm{CI}, 0.7112-0.746, P<$ 0.001 ) demonstrates a strong sensitivity and specificity of our regression model.

In the adjusted analysis for the AIS patients without $\mathrm{AF}$, increasing age $(\mathrm{OR}=0.992,95 \% \mathrm{CI}, 0.986-0.999$, $P=0.032)$, BMI (OR $=0.968,95 \% \mathrm{CI}, 0.955-0.981, \mathrm{P}<$ $0.001)$, depression $(\mathrm{OR}=0.500,95 \% \mathrm{CI}, 0.392-0.639)$, HRT (OR $=0.016,95 \% \mathrm{CI}, 0.001-0.171, P=0.001)$, migraine $(\mathrm{OR}=0.379,95 \% \mathrm{CI}, 0.223-0.642, \mathrm{P}<0.001)$, increasing HDL-cholesterol $(\mathrm{OR}=0.959$, 95\% CI, $0.953-$ $0.966, \mathrm{P}<0.001)$, increasing heart rate $(\mathrm{OR}=0.980,95 \%$ CI, 0.975-0.985, $P<0.001)$, and antihypertensive (OR = $0.810,95 \% \mathrm{CI}, 0.673-0.976, P=0.026)$ were associated with females without $\mathrm{AF}$, while $\mathrm{CAD}(\mathrm{OR}=1.754,95 \%$ CI, 1.450-2.121, $P<0.0001)$, drugs and alcohol $(\mathrm{OR}=$ 3.560, 95\% CI, 2.379-5.327, $\mathrm{P}<0.0001)$, higher serum creatinine level $(\mathrm{OR}=1.218,95 \% \mathrm{CI}, 1.101-1.348, \mathrm{P}<$

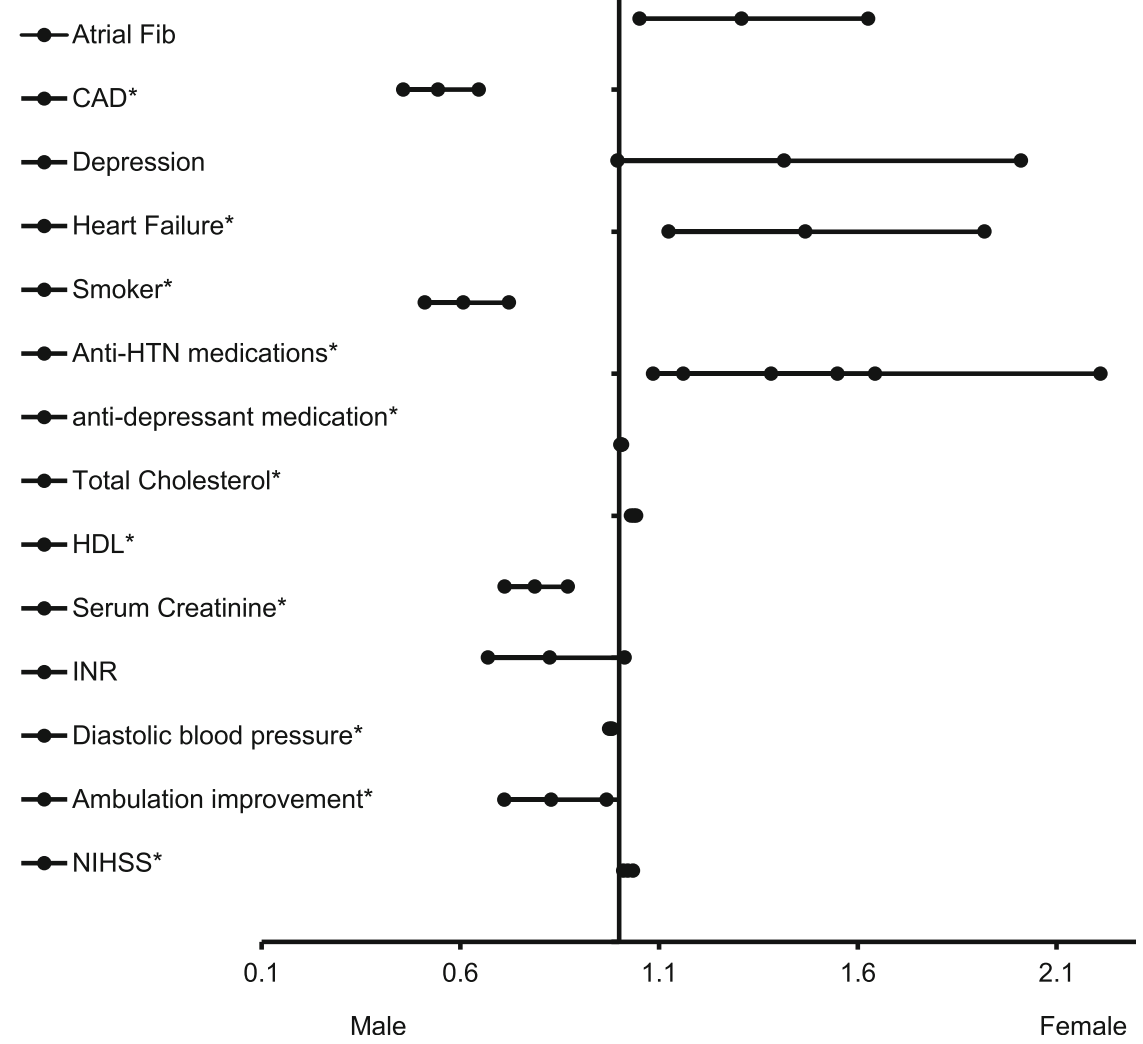

Fig. 1 Forest Plot representation for clinical and demographic factors associated with ischemic stroke patients with and without atrial fibrillation. Adjusted $O R<1$ denotes factors that are associated with male while $O R>1$ denote factors that are associated with females. Hosmer-Lemeshow test $(P=0.546)$, Cox \& Snell $\left(R^{2}=0.149\right)$ were analyzed. The overall classified percentage of $67.1 \%$ was applied to check for fitness of the logistic regression model. *Indicates statistical significance $(P<0.05)$ with a $95 \%$ confidence interval. $\wedge$ Indicates that data were modified by taking the 5 th square root for graphing purposes 


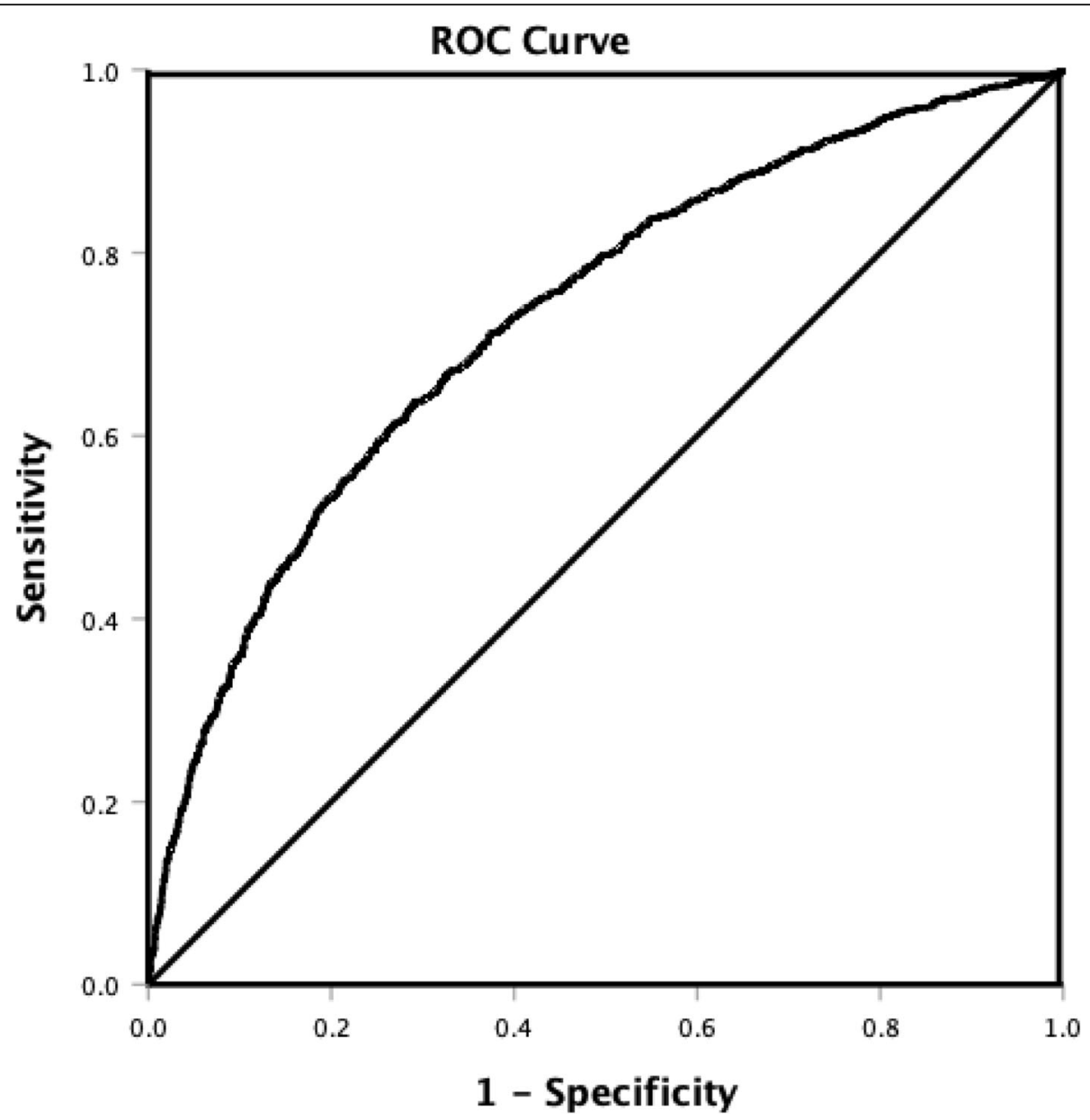

Fig. 2 ROC curve associated with acute ischemic stroke patients with and without atrial fibrillation. Elevated area under the curve (AUC) values in ROC analysis indicate stronger discrimination of the score for being female. ROC curve (AUC $=0.729,0.712-0.746$ ) was used to analyze sensitivity and specificity of the model

$0.0001), \quad \operatorname{INR}(\mathrm{OR}=1.749,95 \% \mathrm{CI}, 1.208-2.532, \quad P=$ $0.003)$, and increasing diastolic blood pressure $(\mathrm{OR}=$ 1.024, 95\% CI, 1.019-1.029, $\mathrm{P}<0.0001$ ), were associated with males (Fig. 3). The ROC Curve (AUC $=0.757$, 95\% CI, 0.740-0.774, $P<0.001)$ demonstrates a strong sensitivity and specificity of the model (Fig. 4).

In the AIS patients with atrial fibrillation (Fig. 5), migraines $(\mathrm{OR}=10.748,95 \% \mathrm{CI}, 0.954-121.135, P=0.016)$, increasing $\mathrm{HDL}(\mathrm{OR}=1.035,95 \% \mathrm{CI}, 1.020-1.050, P<$ 0.001), increasing LDL-cholesterol (1.006, 95\% CI, $1.001-1.011, P=0.003)$, and the inability to ambulate on admission $(\mathrm{OR}=2.258,95 \% \mathrm{CI}, 1.368-3.727, P=0.001)$ were associated with females, while history of drug and alcohol abuse $(\mathrm{OR}=0.250,95 \% \mathrm{CI}, 0.081-0.776, \mathrm{P}=$ $0.016)$, sleep apnea $(\mathrm{OR}=0.321,95 \% \mathrm{CI}, 0.133-0.777$, $P=0.012)$, and increasing serum creatinine level $(\mathrm{OR}=$ $0.693,95 \% \mathrm{CI}, 0.542-0.886, \mathrm{P}=0.003)$ were associated with males. The ROC curve (Fig. 6) demonstrates the sensitivity and specificity of the model to be strong with an AUC of $0.757,95 \%$ CI, 0.721-0.793, and $P<0.001$ ).

\section{Discussion}

Three major findings arise from this study. First, we found that in the whole AIS population with and without AF, coronary artery disease, history of smoking, increasing serum creatinine, increasing diastolic blood pressure, changes or improvement in ambulation, were associated with males, while atrial fibrillation, heart failure, anti-HTN medication, increasing total cholesterol, increasing HDL-cholesterol and increasing NIHSS, were associated with females. Second, in AIS patients without AF, increasing age, BMI, depression, HRT, migraine, increasing HDL-cholesterol, increasing heart rate, and antihypertensive were associated with females without AF, while CAD, drugs and alcohol, higher serum creatinine level, INR, and increasing diastolic blood pressure were associated with males. Finally, in the AIS patients with AF, migraines, increasing HDL, LDLcholesterol, and the inability to ambulate on admission were associated with females, while history of drug and alcohol abuse, sleep apnea, and increasing serum creatinine level were associated with males. 


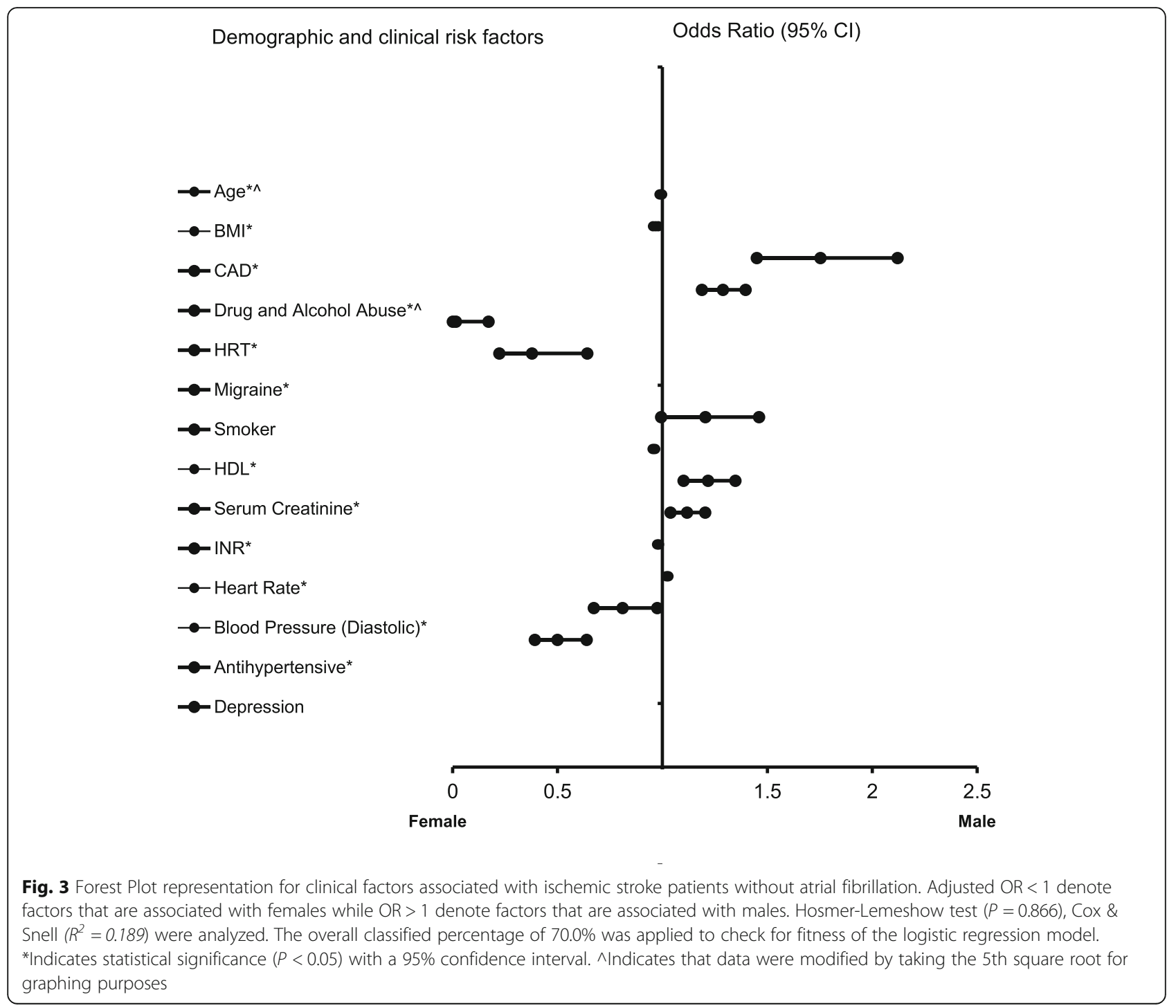

The effect of HDL-cholesterol which was significant in the whole AIS with and without AF was sustained in the adjusted analysis for the female AIS patients without AF. Although age, BMI, depression, HRT, migraine, heart rate, and antihypertensive medications were not associated with female patients in the whole AIS population, these factors were significant and associated with females in the adjusted analysis for the AIS patients without AF. The effect of coronary artery disease, smoking and higher rates of improved ambulation which were more likely to be associated with male patients in the whole AIS with and without AF were attenuated in male patients for the adjusted analysis of AIS patients without AF. In addition, higher serum creatinine levels and diastolic blood pressure were significant among males in the whole AIS with and without AF, and such an effect was sustained and associated with male AIS patients without AF in the adjusted analysis. While CAD, drugs and alcohol were not significant in the whole AIS population, they were significantly associated with males in the adjusted analysis for the AIS without AF.

Our finding that in the AIS population without AF, older AIS patients that present with depression, HRT, migraine, elevated HDL-cholesterol, elevated heart rate, and take antihypertensive medications were associated with females, while CAD, history of drugs and alcohol abuse, high serum creatinine level, elevated INR and increased diastolic blood pressure were associated with males are consistent with other studies for both females [18-21] and for males [22-26]. Findings indicate that female AIS patients present a worse prognosis than men overall [27]. Precisely, hypertension and use of antihypertensive medications were more frequent in females than in males [21, 28]. The effect of depression [29], HRT [30], migraine [31], HDL [32] and heart rate [33] were more severe in females, while CAD [34], drugs and 


\section{ROC Curve}

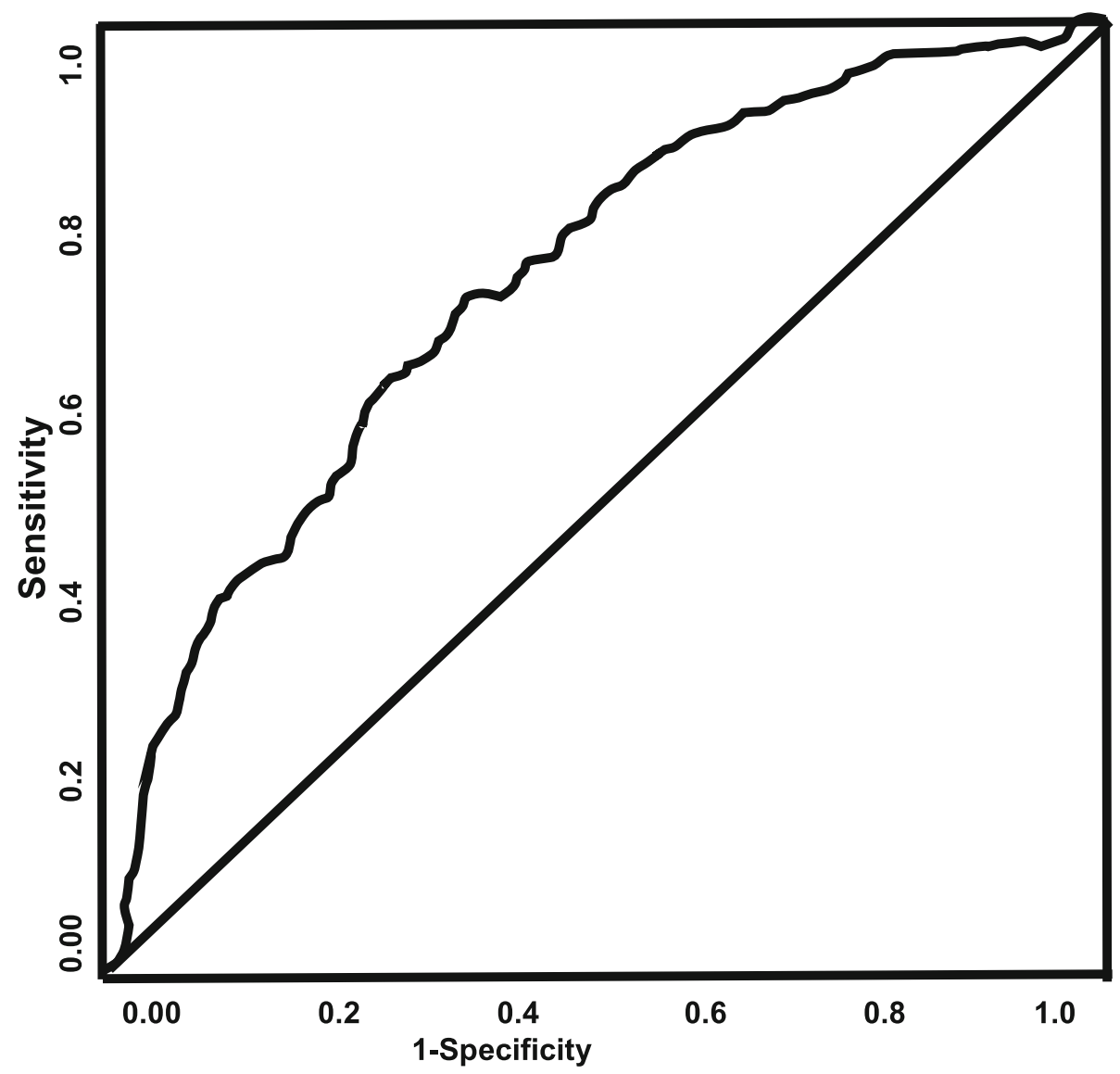

Fig. 4 ROC curve associated with acute ischemic stroke patients without atrial fibrillation. Area under the curve (AUC) values in ROC analysis indicate better discrimination of the score for being male. ROC curve ( $A \cup C=0.757,0.740-0.774$ ) was used to analyze sensitivity and specificity of the model

alcohol use [35], high serum creatinine level [36], INR [25] and diastolic blood pressure [26] were more prevalent in male AIS patients.

We observed that in AIS patients with atrial fibrillation, migraines, elevated HDL-cholesterol and LDLcholesterol with the inability to ambulate on admission were associated with females, while history of drug and alcohol abuse, sleep apnea, and higher serum creatinine level were associated with male AIS patients with AF. Our finding of the association of poor ambulatory outcome with female AIS with AF is consistent with existing evidence on stroke patients [37]. This finding indicates that stroke is not only a leading cause of death, but it is also a leading cause of disability including poor ambulatory outcome particularly in females in whom poor functional outcomes due to a stroke consistently exceeds male patients [38]. Our findings indicate that in AIS patients with AF, excess risk of stroke and poor prognosis may be caused by specific comorbidities and risk factors including migraines, higher HDL-cholesterol, and LDL-cholesterol in female AIS with AF. Migraineurs are known to present with increased levels of cholesterol, HDL-cholesterol, and LDL-cholesterol [39]. In addition, a large population-based cohort study found an increased odd of migraine among those with elevated total cholesterol. This effect was stronger among females that experience migraine than among males [40]. Moreover, females with higher total cholesterol present with an increased risk for AIS compared to females whose cholesterol was lower [31]. Increased risk of stroke is also associated with an elevated ratio of total cholesterol: HDL-cholesterol, or decreased HDL-cholesterol [41]. While previous studies have suggested an increased risk of AIS in males with a high total cholesterol: HDL ratio [42], the current study extends the possibility of HDLcholesterol and LDL-cholesterol to female AIS patients with a baseline AF.

Therefore, our findings that in the AIS patients with $\mathrm{AF}$, migraines, elevated HDL-cholesterol, elevated LDLcholesterol, and poor ambulation were associated with 


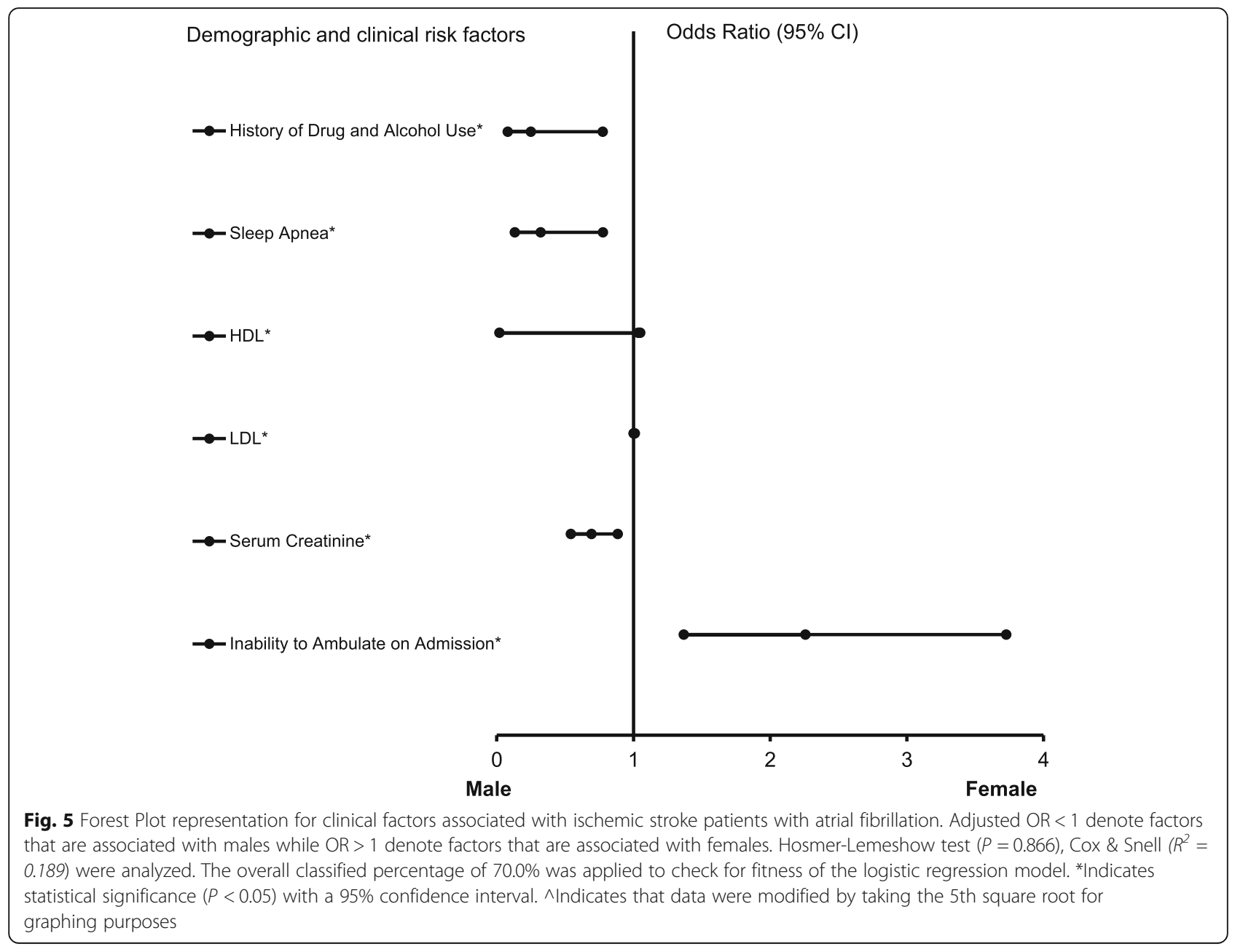

females, while history of drug and alcohol abuse, sleep apnea, and increased serum creatinine level were associated with male AIS patients with AF supports our hypothesis that a gender based disparity in comorbidities and risk factors occurs in AIS population with baseline AF. While the frequencies of vascular disease vary between the males and females AIS patients, and the rates of hypertension (60\% versus $56 \%$ ) are higher in females than in males [33], we show that some risk factors including migraines, HDL-cholesterol, LDL-cholesterol maybe specific to female AIS patients with a baseline AF. Moreover, rates of drugs and alcohol abuse history, sleep apnea, and serum creatinine level were higher in male AIS with baseline AF compared to females.

The prevalence of stroke in the female gender is predicted to rise rapidly due to the continuous increase in the global elderly female population [38]. Our current findings support the possibility that specific baseline risk factors and or comorbidities may have significant effect in females than males, and this may contribute to the observed gender difference among stroke patients with baseline AF. Although our current data does not provide evidence for why a specific comorbidity and or risk factor may have a stronger effect on female AIS with AF than males, there are several possibilities. They include the possibility of under-treatment of females and physiological differences between the male and females [43, 44]. Stroke onset occurs later in age among females compared with males, and rates of $\operatorname{AF}(24 \%$ versus $22 \%)$ are higher in females than in males [45]. Atrial fibrillation is associated with a double the risk of stroke in females compared with the risk in males [43], and females with atrial fibrillation are known to present with more severe strokes than males [46]. Therefore, existing evidence together with our current findings support the possibility that specific comorbidity and or risk factors may have more effect in female than male stroke patients with baseline AF. In this context, the different burden of comorbidities and risk factors in both males and female AIS patients with AF needs to be thoroughly explored to personalize prevention and treatment. 


\section{ROC Curve}

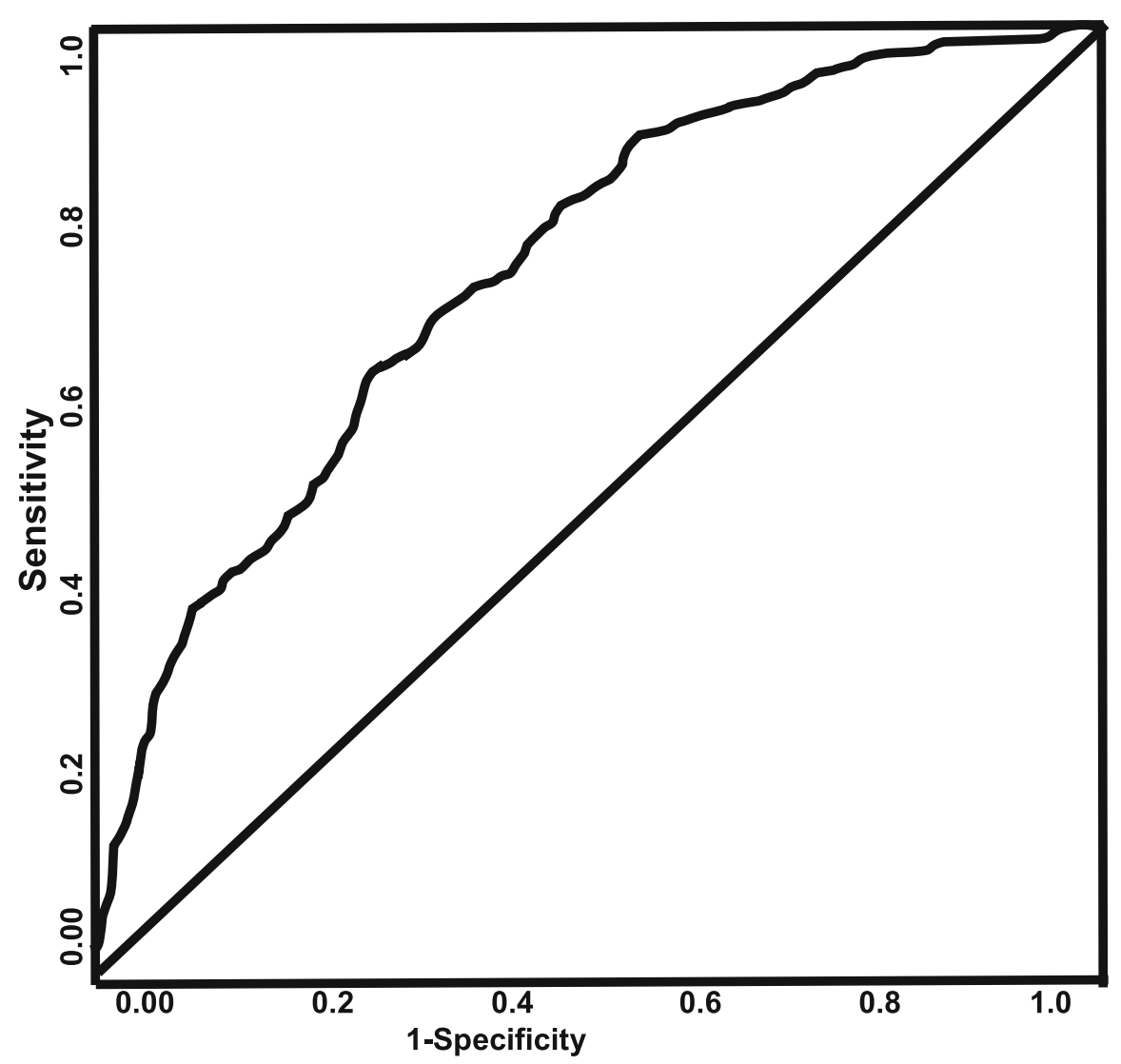

Fig. 6 ROC curve associated with acute ischemic stroke patients with atrial fibrillation. Area under the curve (AUC) values in ROC analysis indicate better discrimination of the score for being female. ROC curve (AUC $=0.757,0.721-0.793$ ) was used to analyze sensitivity and specificity of the model

There some limitations in the interpretation of the results of this study. This study was conducted at a single institution; therefore, the findings cannot be generalized to other institutions. Retrospective studies are known to have biases for selection because the data is not randomized. We predetermined all our subgroup analyses and repeated our analysis several times to eliminate the possibility of type 1 statistical errors. Our female subgroup analyses conducted reveal more significant variables associating more risk factors and comorbidities in females more than that of male AIS with AF. While this is a single study, the demonstration of consistent gender disparities in the AIS with and without AF increases the generalizability of our findings. Since $\mathrm{mRS}$ as a validated index of function for outcome was not included in the registry data based, therefore functional outcome could not be determined.

\section{Conclusion}

Our findings reveal that migraines, elevated HDL, and elevated LDL were associated with female AIS patients with a baseline AF, while drugs and alcohol abuse history, sleep apnea, and higher serum creatinine level were associated with male AIS with baseline AF. Improved management strategies for baseline risk factors for AIS patients with a history of AF will be beneficial for both males and females AIS patients with AF.

\section{Abbreviations}

Adjusted OR: Adjusted odd ratio; Afib: Atrial fibrillation; HRT: Hormone Therapy; BMI: Body mass index; CHF: Congestive heart failure; Cl: Confidence interval; IRB: Institutional Review Board; INR: International normalized ratio; LDL- C: Low-density lipoprotein cholesterol; HDL-C: High-density lipoprotein cholesterol; rtPA: Recombinant tissue plasminogen; TC: Total cholesterol; TG: Triglyceride; AIS: Acute ischemic stroke; NIHSS: National Institute of Health Stroke Scale; MRI: Magnetic Resonance Imaging; CT: Computer Tomography; MCA: Middle cerebral artery; CAD: Coronary artery disease; HRT: Hormone replacement therapy; TIA: Transient ischemic attack; PVD: Peripheral vascular disease; ROC: Receiver Operating Curve; INR: International Normalized Ratio

\section{Acknowledgements}

We thank the stroke unit of PRISMA Health-Upstate for helping in the data collection. 


\section{Authors' contributions}

$\mathrm{CH}, \mathrm{CE}$ and $\mathrm{TN}$ designed the concept, experiment and data analysis, while CBS, KK, and NP critically revised the drafts, interpreted the results, read and approved the last version of this manuscript. The author(s) read and approved the final manuscript.

\section{Funding}

Fullerton Foundation Grant.

\section{Availability of data and materials}

The retrospective datasets are available by request from the corresponding author of this manuscript respectively.

\section{Declarations}

\section{Ethics approval and consent to participate}

This study was performed with the approval of the Institutional Review Board of PRISMA Health System and the institutional Committee for Ethics. The need for informed consent was waived by of PRISMA Health System and the institutional Committee for Ethics. All methods were carried out in accordance with relevant guidelines and regulations.

\section{Consent for publication}

Not applicable.

\section{Competing interests}

None.

Received: 12 January 2021 Accepted: 19 April 2021 Published online: 25 May 2021

\section{References}

1. Chen LY, Chung MK, Allen LA, Ezekowitz M, Furie KL, McCabe P, et al. Atrial fibrillation burden: moving beyond atrial fibrillation as a binary entity a scientific statement from the American Heart Association. Circulation. 2018; 137:E623-E44.

2. Koebe J, Kirchhof P. Novel non-pharmacological approaches for antiarrhythmic therapy of atrial fibrillation. Europace. 2008;10(4):433-7. https://doi.org/10.1093/europace/eun058.

3. Martin RC, Burgin WS, Schabath MB, Kirby B, Chae SH, Fradley MG, et al. Gender-specific differences for risk of disability and death in atrial fibrillation-related stroke. Am J Cardiol. 2017;119(2):256-61. https://doi.org/1 0.1016/j.amjcard.2016.09.049.

4. Marini C, De Santis F, Sacco S, Russo T, Olivieri L, Totaro R, et al. Contribution of atrial fibrillation to incidence and outcome of ischemic stroke - results from a population-based study. Stroke. 2005;36(6):1115-9. https://doi.org/10.1161/01.STR.0000166053.83476.4a.

5. Steger C, Pratter A, Martinek-Bregel M, Avanzini M, Valentin A, Slany J, et al. Stroke patients with atrial fibrillation have a worse prognosis than patients without: data from the Austrian stroke registry. Eur Heart J. 2004;25(19): 1734-40. https://doi.org/10.1016/j.ehj.2004.06.030.

6. Kirchhof P, Benussi S, Kotecha D, Ahlsson A, Atar D, Casadei B, et al. ESC guidelines for the management of atrial fibrillation developed in collaboration with EACTS. Europace. 2016;2016:18.

7. Ko D, Rahman F, Schnabel RB, Yin XY, Benjamin EJ, Christophersen IE. Atrial fibrillation in women: epidemiology, pathophysiology, presentation, and prognosis. Nat Rev Cardiol. 2016;13(6):321-32. https://doi.org/10.1038/nrca rdio.2016.45

8. Lang C, Seyfang L, Ferrari J, Gattringer T, Greisenegger S, Willeit K, et al. Do women with atrial fibrillation experience more severe strokes?: results from the Austrian stroke unit registry. Stroke. 2017;48(3):778-80. https://doi.org/1 0.1161/STROKEAHA.116.015900

9. Ball J, Carrington MJ, McMurray JJV, Stewart S. Atrial fibrillation: profile and burden of an evolving epidemic in the 21st century. Int J Cardiol. 2013; 167(5):1807-24. https://doi.org/10.1016/j.ijcard.2012.12.093.

10. Saad EB, Tayar DO, Ribeiro RA, Junqueira SM, Andrade P, d'Avila A Healthcare utilization and costs reduction after radiofrequency ablation for atrial fibrillation in the Brazilian private healthcare system. Arq Bras Cardiol. 2019;113:252-6.

11. Gedikli O, Mohanty S, Trivedi C, Gianni C, Chen Q, Della Rocca DG, et al. Impact of dense "smoke" detected on transesophageal echocardiography on stroke risk in patients with atrial fibrillation undergoing catheter ablation. Heart Rhythm. 2019;16(3):351-7. https://doi.org/10.1016/j.hrthm.2018.10.004.

12. Feng $S D$, Jiang $Y$, Lin $Z H$, Guo $P Q, W u ~ Y L$, Ling PH. Influence of atrial fibrillation on prognosis of thrombolytic therapy in stroke patients: a metaanalysis. Int J Clin Exp Med. 2016;9:9203-10.

13. Appleton S, Gill T, Taylor A, McEvoy D, Shi ZM, Hill C, et al. Influence of gender on associations of obstructive sleep apnea symptoms with chronic conditions and Quality of life. Int J Environ Res Public Health. 2018;15(5). https://doi.org/10.3390/ijerph15050930.

14. Asplund K, Tuomilehto J, Stegmayr B, Wester PO, Tunstallpedoe H. Diagnostic-criteria and quality-control of the registration of stroke events in the MONICA project. Acta Medica Scandinavica. 1988;728:26-39.

15. Brecthel L, Gainey J, Penwell A, Nathaniel TI. Predictors of thrombolysis in the telestroke and non telestroke settings for hypertensive acute ischemic stroke patients. BMC Neurol. 2018;18(1):215. https://doi.org/10.1186/s12883-018-1204-3.

16. Lawson TR, Brown IE, Westerkam DL, Blackhurst DW, Sternberg S, Leacock R, et al. Tissue plasminogen activator (rt-PA) in acute ischemic stroke: outcomes associated with ambulation. Restor Neurol Neurosci. 2015;33(3): 301-8. https://doi.org/10.3233/RNN-140480.

17. Nathaniel TI, Gainey J, Blum B, Montgomery C, Ervin L, Madeline L. Clinical risk factors in thrombolysis therapy: Telestroke versus Nontelestroke. J Stroke Cerebrovasc Dis. 2018;27(9):2524-33. https://doi.org/10.1016/j. jstrokecerebrovasdis.2018.05.012.

18. Sacco S, Ricci S, Degan D, Carolei A. Migraine in women: the role of hormones and their impact on vascular diseases. J Headache Pain. 2012; 13(3):177-89. https://doi.org/10.1007/s10194-012-0424-y.

19. Kurth T, Everett BM, Buring JE, Kase CS, Ridker PM, Gaziano JM. Lipid levels and the risk of ischemic stroke in women. Neurology. 2007;68(8):556-62. https://doi.org/10.1212/01.wnl.0000254472.41810.0d.

20. Hsia J, Larson JC, Ockene J, Sarto GE, Allison MA, Hendrix S, et al. Resting Heart Rate Predicts Coronary Events, but not Stroke, in Women. Circulation. 2008:118:S1126-S.

21. Gainey J, Brechtel L, Konklin S, Madeline L, Lowther E, Blum B, et al. In a stroke cohort with incident hypertension; are more women than men likely to be excluded from recombinant tissue-type plasminogen activator (rtPA)? J Neurol Sci. 2018;387:139-46. https://doi.org/10.1016/j.jns.2018.02.016.

22. Sato C, Wakabayashi K, Ikeda N, Honda Y, Sato K, Suzuki T, et al. Influence of sex on the incidence of potential coronary artery disease and long-term outcomes in asymptomatic patients with diabetes mellitus. IJC Heart Vasc. 2020;27:100504. https://doi.org/10.1016/j.ijcha.2020.100504.

23. de los Rios F, Kleindorfer DO, Khoury J, Broderick JP, Moomaw CJ, Adeoye $\mathrm{O}$, et al. Trends in Substance Abuse Preceding Stroke Among Young Adults A Population-Based Study. Stroke. 2012;43:3179-83.

24. Ibrahim B, Rayyis L, Almekhlafi M. Elevated serum creatinine predicts higher mortality in stroke patients. Neurology. 2017:88:254-6.

25. Yamaguchi T, Japanese Nonvalvular Atrial F. Optimal intensity of warfarin therapy for secondary prevention of stroke in patients with nonvalvular atrial fibrillation - A multicenter, prospective, randomized trial. Stroke. 2000; 31:817-21.

26. Park J, Ovbiagele B. Post-stroke diastolic blood pressure and risk of recurren vascular events. Stroke. 2018:49(Suppl_1). https://doi.org/10.1161/str.49. suppl_1.WP391.

27. Arboix A, Cartanya A, Lowak M, Garcia-Eroles L, Parra O, Oliveres M, et al. Gender differences and woman-specific trends in acute stroke: results from a hospital-based registry (1986-2009). Clin Neurol Neurosurg. 2014;127:1924. https://doi.org/10.1016/j.clineuro.2014.09.024

28. Fleming T, Blum B, Averkamp B, Sullivan J, Nathaniel T. Effect of antihypertensive medications on thrombolysis therapy and outcomes in acute ischemic stroke patients. J Clin Hypertens. 2019;21(2):271-9. https://doi.org/10.1111/jch.13472

29. Albert PR. Why is depression more prevalent in women? J Psychiatry Neurosci. 2015;40(4):219-21. https://doi.org/10.1503/jpn.150205.

30. Bolton JL. Menopausal hormone therapy, age, and chronic diseases: perspectives on statistical trends. Chem Res Toxicol. 2016;29(10):1583-90. https://doi.org/10.1021/acs.chemrestox.6b00272.

31. Kurth T, Rist PM, Ridker PM, Kotler G, Bubes V, Buring JE. Association of Migraine with Aura and Other Risk Factors with Incident Cardiovascular Disease in women. JAMA. 2020;323(22):2281-9. https://doi.org/10.1001/ja ma.2020.7172.

32. Fernandez ML, Herron K, Ramjiganesh T, Roy S, Vega-Lopez S. Eggs raise plasma total and HDL cholesterol in pre-menopausal women without affecting total/HDL cholesterol ratios. Faseb J. 2001;15:A274-A. 
33. Matsumoto Y, Fukushima S, Shimahara Y, Tadokoro N, Kakuta T, Kobayashi J, et al. Sex differences in continuous-flow ventricular assist device therapy for advanced heart failure. Gen Thorac Cardiovasc Surg. 2021;69:919-25.

34. Iwasaki K, Haraoka K, Hamaguchi T, Imamura T, Kawada S, Ohno M, et al. Prevalence of subclinical coronary artery disease in ischemic stroke patients. J Cardiol. 2015;65(1):71-5. https://doi.org/10.1016/j.jjcc.2014.04.004.

35. Rotimi OR, Ajani IF, Penwell A, Lari S, Walker B, Nathaniel TI. In acute ischemic stroke patients with smoking incidence, are more women than men more likely to be included or excluded from thrombolysis therapy? Womens Health. 2020;16:174550652092276. https://doi.org/10.1177/174 5506520922760

36. Snarska K, Kapica-Topczewska K, Bachorzewska-Gajewska H, Malyszko J. Renal function predicts outcomes in patients with Ischaemic stroke and Haemorrhagic stroke. Kidney Blood Press Res. 2016;41(4):424-33. https://doi. org/10.1159/000443444

37. Scalise M, Brechtel L, Conn Z, Bailes B, Gainey J, Nathaniel IT. Predicting ambulatory recovery in acute ischemic stroke patients with thrombolytic therapy. Future Neurol. 2020;4:34-9.

38. Cordonnier C, Sprigg N, Sandset EC, Pavlovic A, Sunnerhagen KS, Caso V, et al. Stroke in women - from evidence to inequalities. Nat Rev Neurol. 2017;13(9):521-32. https://doi.org/10.1038/nrneurol.2017.95.

39. Gruber HJ, Bernecker C, Pailer S, Lechner A, Horejsi R, Moller R, et al. Lipid profile in normal weight migraineurs - evidence for cardiovascular risk. Eur J Neurol. 2010;17(3):419-25. https://doi.org/10.1111/j.1468-1331.2009.02861.x.

40. Ikeda K, Kashihara H, Hosozawa Kl, Maruyama Y, Tamura M, Iwasaki Y. Cardiovascular risk factors and migraine: The GEM population-based study. Neurology. 2005;65:1683.

41. Poupore N, Strat D, Mackey T, Brown K, Snell A, Nathaniel TI. Cholesterol reducer and thrombolytic therapy in acute ischemic stroke patients. Lipids Health Dis. 2020;19(1):84. https://doi.org/10.1186/s12944-020-01270-2.

42. Wannamethee SG, Shaper AG, Ebrahim S. HDL-cholesterol, total cholesterol, and the risk of stroke in middle-aged British men. Stroke. 2000;31(8):1882-8. https://doi.org/10.1161/01.STR.31.8.1882.

43. Emdin CA, Wong CX, Hsiao AJ, Altman DG, Peters SAE, Woodward M, et al. Atrial fibrillation as risk factor for cardiovascular disease and death in women compared with men: systematic review and meta-analysis of cohort studies. BMJ. 2016;352:h7013.

44. Peters SAE, Huxley RR, Sattar N, Woodward M. Sex differences in the excess risk of cardiovascular diseases associated with type 2 diabetes: potential explanations and clinical implications. Curr Cardiovasc Risk Rep. 2015;9(7):36. https://doi.org/10.1007/s12170-015-0462-5.

45. Peters SAE, Huxley RR, Woodward M. Do smoking habits differ between women and men in contemporary Western populations? Evidence from half a million people in the UK biobank study. BMJ Open. 2014;4(12): e005663. https://doi.org/10.1136/bmjopen-2014-005663.

46. Nezu T, Hosomi N, Kondo K, Aoki S, Matsumoto M, Kobayashi S, et al. Greater Severity of Neurological Defects in Women Admitted With Atrial Fibrillation-Related Stroke. Circ J. 2015;80:250-5.

\section{Publisher's Note}

Springer Nature remains neutral with regard to jurisdictional claims in published maps and institutional affiliations.

Ready to submit your research? Choose BMC and benefit from:

- fast, convenient online submission

- thorough peer review by experienced researchers in your field

- rapid publication on acceptance

- support for research data, including large and complex data types

- gold Open Access which fosters wider collaboration and increased citations

- maximum visibility for your research: over $100 \mathrm{M}$ website views per year

At $\mathrm{BMC}$, research is always in progress.

Learn more biomedcentral.com/submissions 